\title{
La inclusión de las mujeres en las clases de historia: posibilidades y limitaciones desde las concepciones de los estudiantes chilenos
}

Women Inclusion in History Class: Possibilities and Limitations from Chilean Students' Conceptions

A inclusão das mulheres nas aulas de história: possibilidades e limitações desde as concepções dos estudantes chilenos

Jesús Marolla* iD orcid.org/0000-0001-6215-0010

\section{(c) $\underset{\mathrm{BY}(\mathrm{NC})}{(-)}$}




\section{Resumen}

El presente artículo es resultado de una investigación que indaga las concepciones de los estudiantes chilenos sobre la inclusión y la ausencia de las mujeres en la enseñanza de la historia y las ciencias sociales. Los objetivos perseguidos se resumen en la comprensión de las posibilidades y los obstáculos que plantean los alumnos para poder trabajar con las mujeres y su historia desde perspectivas críticas y reflexivas. La metodología planteada es un estudio de casos llevado a cabo a través de grupos focales con distintos jóvenes de escuelas de Santiago de Chile. Los lineamientos metodológicos se enmarcan en el análisis cualitativo y las teorías feministas de investigación. De esa manera, se plantea el análisis de los resultados desde la teoría crítica y la teoría queer como ejes para comprender lo que plantean los estudiantes. Entre los principales resultados es posible comentar que los jóvenes afirman que las mujeres sí tienen historia, no obstante, ha sido omitida y marginada por las decisiones y acciones de los hombres a fin de generar jerarquías donde ellos se mantienen en los espacios de poder. Los alumnos agregan que se debe problematizar la ausencia y los roles de las mujeres desde perspectivas de género y desde la didáctica a fin de romper con las desigualdades sociales.

\section{Palabras clave}

mujeres; género; inclusión; enseñanza de la historia; estudiantes

\section{Keywords}

women; gender; inclusion; teaching history; students

\begin{abstract}
This article is the result of an investigation about the conceptions of Chilean students on the inclusion and absence of women in the teaching of history and socia sciences. The goals of the study can be summarized into understanding the possibilities and the obstacles raised by the students to work with women and their history from critical and reflective perspectives. The methodology is a case study carried out through focus groups with different students from schools in Santiago, Chile. The methodological guidelines are framed in qualitative analysis and feminist research theories. Thus, the paper suggests analyzing the results from the point of view of critical theory and queer theory. Among the main results, we can say that students consider that women do have a history, but that it has been omitted and marginalized by the decisions and actions of the men in order to generate hierarchies where they keep the power in society. The students add that the absence and roles of women must be problematized from gender perspectives and from didactics in order to end social inequalities.
\end{abstract}

\section{Resumo}

Este artigo é o resultado de uma investigação que explora os conceitos de estudantes chilenos e sobre a inclusão e a ausência de mulheres no ensino de História e Ciências Sociais. Os objetivos são resumidos na compreensão das possibilidades e obstáculos colocados pelos estudantes para poder trabalhar com mulheres e sua história a partir de perspectivas críticas e reflexivas. A metodologia proposta é um estudo de caso realizado por meio de grupos focais com diversos jovens e diferentes escolas de Santiago de Chile. As orientações metodológicas são parte da análise qualitativa e as teorias feministas de pesquisa. Dessa forma, a análise dos resultados baseia-se na teoria crítica e na teoria queer como eixos para entender o que os alunos afirmam. Os principais resultados assinalam que os jovens afirmam que as mulheres têm história, no entanto, foi omitida e marginalizadas pelas decisões e ações dos homens, a fim de gerar hierarquias nas que eles permanecem nas posições de poder. Os estudantes afirmam que é necessário problematizar a ausência e os papéis das mulheres desde perspectivas de gênero e desde a didática, a fim de eliminar as desigualdades sociais.

\section{Palavras-chave}

mulheres; gênero; inclusão ensino da história; estudantes 


\section{Introducción}

En la actualidad, la inclusión de la diversidad de géneros resulta relevante y un reto para las escuelas, la enseñanza y la didáctica de las ciencias sociales. Esto lo evidencian estudios realizados desde áreas como el currículo y la psicología del aprendizaje (Crocco, 2008; Fernández, 2006; Levstik y Groth, 2002; Lerner, 1979; Mcintosh, 1983; Woyshner, 2002). Desde algunas teorías de género se ha puesto el foco en la educación (Britzman, 2002; Talburt y Steinberg, 2005) a fin de comprender cómo las escuelas producen y reproducen identidades y se consolidan los binarios de normal/anormal, hombre/mujer. Estos estudios coinciden en afirmar que este tratamiento es una de las formas en que se establecen las estructuras de inclusión/exclusión en torno a las normas de género tradicionales.

Algunos estudios desde la didáctica de las ciencias sociales (Fernández, 2006; Marolla y Pagès, 2015; Pagès y Sant, 2012), se han preocupado por los currículos, los programas, la formación y el trabajo que realiza el profesorado desde la inclusión de los invisibles en la enseñanza de la historia y las ciencias sociales. En especial, se han interesado por la presencia/ausencia de las mujeres en el currículum, los programas y los libros de texto.

Los objetivos planteados en esta investigación buscan reflexionar sobre el contenido de los discursos de distintos estudiantes chilenos sobre la inclusión y el trabajo con las mujeres en las clases de historia. Esto se interpretará desde las posibilidades, las ventajas y los obstáculos que comentan los estudiantes para generar cambios ante las desigualdades que ocurren en las aulas en torno a la ausencia de las mujeres y de su historia.

\section{Los conceptos de mujeres, género e identidades en la investigación}

Para la investigación resulta fundamental comprender y adoptar un posicionamiento ante los conceptos de mujer y de género. Tal posicionamiento se define desde perspectivas feministas posestructuralistas (Butler, 2001). Mouffe (1993) afirma que el género y el concepto de mujer no responden a construcciones permanentes, sino que está en constante proceso de producción-reproducción. Las concepciones de mujer, género e identidad se exponen a continuación:

a. Mujeres. Comprender y problematizar el concepto de mujer es una manera de reflexionar sobre cómo se han construido y cómo actúan las jerarquías de la diferencia, las inclusiones y las exclusiones. La problematización de las mujeres como grupo no busca, en esencia, conocer su historia como narrativa, sino comprender cómo se han construido las 
categorías subjetivas y las estructuras de dominación y de subordinación hacia las mujeres (Scott, 2008), lo que, por tanto, ha provocado su marginación e invisibilización.

El objetivo de tales perspectivas, siguiendo a Butler (2001), es deconstruir los conceptos para construir nuevas categorías que no sean sólidas, ni que actúen con un objetivo normalizador desde el poder (Foucault, 2008). Los planteamientos anteriores buscan crear marcos de inclusión donde se respete a las personas, se reconozca la vida humana y, en especial, las subordinaciones en que se han relegado a las mujeres, y así se abran espacios para una ciudadanía dinámica donde el diálogo y la democracia sean los fundamentos.

b. Género. Según Scott (2008), significa conocimiento de la diferencia sexual. Para Foucault (2008) es una producción social creada entre las relaciones de poder, de dominación y de subordinación. Scott (2008) no se refiere a que el género instaura las diferencias físicas entre mujeres y hombres, sino que el género es un conocimiento que ha sido producido por epistemologías históricas, creado a través de discursos que han establecido un sistema de jerarquías, inclusiones y exclusiones binarias basadas en los rasgos físicos de las personas.

c. Identidades. Butler (2001) y Foucault (2008) plantean que la identidad siempre se construye dentro de complejas interacciones entre el discurso y el poder, y este se entiende en función de las convenciones culturales heterosexuales y fálicas. En relación con lo anterior, Mouffe (1993) afirma que no es posible existir en el género de manera total y única, ya que no existen las identidades sociales completas y permanentes. Así las identidades son parte de múltiples y constantes cambios.

Siguiendo Wittig (2006), se ha definido que tanto la biología como la naturaleza no conforman al hombre ni a la mujer, sino que la existencia de los binarios responde a estructuras simbólicas ligadas de forma dialéctica con apariencia de solidez e inamovilidad. La mujer solo existiría en los sistemas heterosexuales de pensamiento que la relacionan con un opuesto que la define y la construye (Wittig, 2006).

Scott (2008), al respecto, plantea que la historia se ha escrito excluyendo a las mujeres y a los estudios de género debido a que la cultura ha sido patriarcal. La historia se ha configurado, en este sentido, desde la masculinidad. Tal masculinidad configura sus símbolos y prácticas desde la exclusión de las mujeres (Butler, 2006). De ahí que han existido diversas expresiones históricas que han perseguido, por ejemplo, visibilizar a las mujeres en igualdad a las acciones de los hombres, destacando la represión, la marginación, la ausencia y las jerarquías por razones de género. Los objetivos de tales perspectivas, además de denunciar las opresiones y reflexionar sobre los caminos a seguir para la deconstrucción de las posiciones subordinadas 
de las mujeres (y de otros géneros), se ha planteado como fundamental la necesidad de la creación de nuevas periodizaciones y narrativas desde y para la construcción de la historicidad femenina (Scott, 2008).

Según Scott (2008), la historia de las mujeres, la historia feminista o la historia de los géneros no se debe remitir al recuento de las grandes obras realizadas por las mujeres, sino a la exposición de aquellas acciones silenciadas del género. La autora reconoce la importancia de rescatar las acciones de las mujeres en la construcción de las narrativas históricas; no obstante, afirma que la reflexión debe orientarse hacia el empoderamiento de las mujeres ante las desigualdades por razones de género.

\section{La inclusión/exclusión de las mujeres en la enseñanza de la historia}

Crocco (2008) comenta que los estudios de género y la inclusión de las mujeres en la enseñanza podrían conllevar un cambio tanto a nivel del discurso, como de lo que se entiende por la enseñanza para la ciudadanía. Fernández (2006) coincide con la autora en que la inclusión de las mujeres podría provocar que se reflexionara sobre quiénes han sido los protagonistas de la historia.

Subirats (2001) afirma la urgencia de construir una enseñanza que fomente la diversidad de género. Para ello es necesario reinterpretar la historia y establecer un espacio en donde se deje en evidencia el rol que han cumplido tanto las mujeres, como el resto de personas que habitan el mundo y que han sido silenciadas por una historia que ha dotado de protagonismo a los hombres con poder por sobre los demás.

Autores como Hubbard (2013), Subirats (2001) y Vázquez (2003) plantean que la escuela se ha construido bajo los lineamientos del patriarcado. El androcentrismo transmite fuertes estereotipos y prejuicios que, directa e indirectamente, marginan y segregan a las mujeres por razones de género. Se debe repensar y subvertir la construcción de la escuela con el objetivo de crear espacios de igualdad y de diferencia y de múltiples identidades y sexualidades.

\section{La inclusión de las mujeres en los libros de texto}

Autores como Crocco (2010) y Pagès y Sant (2012) coinciden en que los libros se han construido y se apoyan, sobre todo, en el protagonismo de los hombres y el de masas anónimas que se presentan de manera masculinizada. Lomas (2002) afirma que los libros traducen y replican el androcentrismo que existe en la sociedad. La imagen que se ofrece 
sobre las mujeres, agrega, es la de un personaje relegado eminentemente al ámbito doméstico y privado, sin mayor participación en la sociedad ni en la construcción de la historia.

Los trabajos de Levstik y Barton (1997) y Levstik y Groth (2002) argumentan que los protagonistas de los textos son aquellos hombres ligados al poder, la administración, la política y la esfera pública. Según estos autores, la exclusión y la invisibilidad de las mujeres provoca que la enseñanza genere estereotipos y prejuicios en torno a ellas y a su participación en la historia. De esta manera, las mujeres y su historia están ausentes de las narrativas, y cuando se hacen presentes, se realiza desde concepciones sesgadas que provocan su marginación en la sociedad.

\section{Estudios sobre el profesorado y la inclusión de las mujeres}

Hubbard (2013) y Vázquez (2003) coinciden en plantear que ni la formación ni las prácticas que realiza el profesorado consideran las problemáticas de las mujeres. Crocco (2010) y Woyshner (2002) acuerdan que el profesorado actúa como un agente en la transmisión de los estereotipos, los prejuicios y las desigualdades de género. Moreno y Sastre (2003) afirman que esto se debe a que el profesorado tiende a enseñar desde el modelo "más valorado socialmente", o sea, desde lo masculino.

Crocco (2010) considera que debe existir un cambio de enfoques en las prácticas del profesorado y en el proceso de enseñanza y aprendizaje que se está realizando. Al respecto, Heimberg (2005) cree que se deberían potenciar discursos y prácticas hacia la inclusión, la empatía y los espacios de empoderamiento a través de la identificación con la historia.

Por último, para Crocco (2006) y Banks (2004), la mayoría de los estudiantes seguirán estando marginados mientras las mujeres, las minorías culturales, los trabajadores, entre otros colectivos, sean excluidos en función de los valores y las experiencias de los grupos dominantes. Para el caso de la investigación los grupos dominantes son representados por aquellos hombres blancos y con poder político, económico o militar (Levstik y Barton, 1997). Vázquez (2003) agrega que si el profesorado no adopta nuevas perspectivas para incluir a las mujeres en las clases, se continuarán reproduciendo los estereotipos y los prejuicios por razones de género.

\section{Metodología}

El escrito se posiciona en el paradigma cualitativo a fin comprender y dar relevancia y complejidad a los problemas sociales (Álvarez-Gayou, 2003; Creswell, 2014; Simons, 2011; Stake, 2007). Dentro de tal paradigma, 
para Álvarez-Gayou (2003) la preocupación del escrito se inserta en la reflexión y comprensión de los problemas por razones de género que han experimentado las mujeres y aquellos excluidos de la enseñanza de la historia y las ciencias sociales.

Se utiliza tanto la teoría crítica (Álvarez-Gayou, 2003; Cohen, Manion y Morrison, 2007), como las aportaciones de la teoría de investigación feminista (Cohen, Manion y Morrison, 2007; Usher, 1996). Estas investigaciones destacan por potenciar la voz, la emancipación y la igualdad de los grupos oprimidos, y pretenden transformar las jerarquías y las estructuras de poder que han marginado y silenciado a las mujeres por razones de género. La investigación se sitúa en aspecto como la explotación y la opresión hacia las mujeres desde perspectivas de género.

El enfoque es un estudio de casos construido siguiendo los planteamientos de Simons (2011) y Stake (2007). Se usa este enfoque debido a que el objeto de estudio está centrado en un fenómeno o problema social (Simons, 2011). Según Cohen, Manion y Morrison (2007), el enfoque es un estudio de casos exploratorio (Yin, 2009) e interpretativo (Simons, 2011).

Siguiendo a Cohen, Manion y Morrison (2007); Simons (2011), y Stake (2007), los estudios de casos son útiles cuando se investigan problemas sociales complejos ya que permiten: 1 ) descubrir hechos o procesos sutiles; 2) otorgar posibilidades para desvelar significados profundos y desconocidos; 3 ) informar y colaborar en la comprensión de realidades educativas complejas; 4) utilizar distintas técnicas de recolección y análisis de datos; 5) implicar a los participantes y al investigador en un proceso de comprensión sobre las prácticas.

Sin embargo, los estudios de caso tienen también inconvenientes como los siguientes: 1) dificultad para procesar la gran cantidad de datos acumulados; 2) subjetividad del investigador y su modo de inferir en los casos, y 3) validez y utilidad de las conclusiones. No obstante, se han sorteado tales inconvenientes a fin de asegurar la validez del análisis así como las perspectivas que se plantean desde las posibilidades y los obstáculos para la inclusión de las mujeres y su historia en la enseñanza.

\section{El diseño de la investigación}

El análisis cualitativo de la información se ha realizado a través de la síntesis de los planteamientos de Creswell (2014) en la organización y la creación de códigos y Stake (2007), Simons (2011) y Miles y Huberman (1994) para la reducción, las conclusiones y la verificación de los datos. En el estudio resultan fundamentales los planteamientos de Lather (1992), 
Habermas (1988), Finlay (2002), Macbeth (2001) y Oakley (1988) para reflexionar y plantear la deconstrucción de contenidos de los discursos de los estudiantes. El diseño de la investigación ha seguido las siguientes fases:

a. Fase de reducción. Se redujeron y seleccionaron los principales aspectos manifestados en los grupos focales y que respondieran a los objetivos de la investigación.

b. Fase analítica. Se organizaron los datos y se crearon las primeras categorías a fin de responder a las preguntas y los objetivos planteados.

c. Fase interpretativa de casos. Siguiendo a Bardin (1986) y el análisis cualitativo de contenido, se organizaron los datos y se establecieron patrones y esquemas a fin de comprender y reflexionar sobre el problema planteado. En tal fase se procedió a la triangulación de la información. Para llevar a cabo este proceso se usaron dos estrategias:

» Suma categórica (Stake, 2007). Se establecieron los patrones, conceptos y se clasificaron los datos en categorías que respondieran a los contenidos.

» Categorización y codificación, donde desde la categorización realizada se procedió a definir las categorías de contenido (Miles y Huberman, 1994).

d. Fase ideológica interpretativa. Se establecieron dos etapas.

La primera es la discursiva para la emancipación. Aquí se llevaron a cabo las interpretaciones y las explicaciones sobre los factores que intervienen en la problemática. De la misma manera se plantearon las opciones de transformación que existen para la justicia social de las personas y en especial desde la subordinación de las mujeres (Habermas, 1988; Lather, 1992; Wolcott, 1994).

Una segunda fase para la deconstrucción, en la cual se generaron los planteamientos para la emancipación de las mujeres desde las problemáticas de género enfocadas en la enseñanza de la historia y las ciencias sociales. Tales planteamientos son generados desde el análisis de los discursos desde la teoría crítica (Habermas, 1988; Lather, 1992), la teoría feminista (Finlay, 2002; Macbeth, 2001) y la justicia social (Finlay, 2002; Habermas, 1988; Macbeth, 2001).

\section{Los grupos focales}

La selección de tal método responde a las posibilidades de informar a las preguntas de investigación (Simons, 2011). Los grupos focales se enmarcan en la teoría crítica (Habermas, 1988) y plantean una finalidad de emancipación y de empoderamiento para el cambio social. 
La elección de los grupos focales, según Álvarez-Gayou (2003), Bisquerra (2004) y Cohen, Manion y Morrison (2007), se ha realizado debido a que permiten reunir una gran cantidad de datos sobre un tema en particular, además de las interacciones y las discusiones que pueden generar los participantes sobre el tópico de la investigación y el problema del estudio (Álvarez-Gayou, 2003).

El diseño de los grupos focales se ha realizado siguiendo los criterios de Álvarez-Gayou (2003), Bisquerra (2004) y Cohen, Manion y Morrison (2007): 1) número de grupos y de participantes; 2) selección, duración, guía y encuadre técnico, y 3 ) informantes dispuestos a compartir sus experiencias. Estos criterios colaboraron en que el estudio se enmarcara en dar respuesta a las preguntas y los objetivos planteados.

\section{Participantes de los casos}

Se han realizado cuatro grupos focales. Los participantes son alumnos y alumnas de distintas escuelas de Santiago de Chile. Los criterios utilizados para la elección, siguiendo a Bisquerra (2004), son: 1) facilidad para acceder y permanecer en el campo; 2) posibilidad de asegurar la calidad y la credibilidad del estudio; 3) ubicación geográfica, y 4) disposición de las instituciones y de las personas a participar.

Siguiendo los criterios de Simons (2011) y Stake (2007), se escogieron tales participantes no con una finalidad de representatividad. La elección se debió a las posibilidades que ofrecían para explicar las diferencias en las concepciones de cada estudiante frente a las prácticas y la inclusión de las mujeres y su historia, y de esa manera, comprender la problemática desde distintas perspectivas.

Según los planteamientos de Stake (2007) y Simons (2011) para la elección de los casos se consideraron tres criterios: 1) la elección intencional, donde se seleccionaron algunos estudiantes que estuvieran dispuestos a compartir sus experiencias en los grupos focales; 2 ) el equilibrio y la variedad de los participantes, y 3) el tipo de escuela (pública, privada, subvencionada ${ }^{1}$ ). Los alumnos participantes se relacionan en la tabla 1.

1 El modelo subvencionado en Chile recibe fondos del Estado y además un monto fijo mensual o anual por las personas que están a cargo del estudiante matriculado. 
Tabla 1

Alumnos participantes en los grupos focales

\begin{tabular}{ccccc}
\hline Profesor/a & Alumnos(as) & Curso & Edad & Fecha grupo focal \\
\hline Paula & Mauricio, Richard, Dania, Leo, Cony. & $8^{\circ}$ básico & $13-14$ años & 4-12-2014 \\
Marcos & Samuel, Jorge, Juan, Fabián, Arturo. & $2^{\circ}$ medio & $15-16$ años & $11-12-2014$ \\
David & Fany, Irma, Adriana, Camila. & $3^{\circ}$ medio & $16-17$ años & 9-12-2014 \\
Luis & Amelia, Gabriel, Pato, Violeta. & $8^{\circ}$ básico & 13-14 años & 16-12-2014 \\
\hline
\end{tabular}

Fuente: elaboración propia.

\section{Resultados de la investigación}

Para la investigación resultan fundamentales ya que permiten conocer y reflexionar sobre qué concepciones construyeron sobre la presencia/ ausencia de las mujeres en la enseñanza.

\section{La presencia o ausencia de las mujeres en las clases de historia y ciencias sociales}

Los grupos de Luis, Paula y Marcos afirman y coinciden en que trabajan temas como la política, las guerras, la economía y la filosofía principalmente. Por otra parte, las alumnas del grupo de David destacan sus preferencias por temáticas como la vida laboral, la vida privada, los trabajos de los campesinos, los problemas de las mujeres, entre otros pertenecientes a la historia social. No obstante, esos aspectos que se trabajan, agregan, son esporádicos en la enseñanza de la historia. En este sentido, coinciden en que los temas son parte de la periodización tradicional de la historia (prehistoria, historia antigua, historia medieval, historia moderna).

Samuel, del grupo de Marcos, afirma que los temas políticos son el centro de las clases: "Este año ha sido más político [...]. Grecia y Roma, tratamos sobre la mitología y la forma de gobernar de los griegos y los romanos [...]". Arturo agrega que se han fomentado temas desde la política donde se destacan las acciones de los hombres. El estudiante dice que se ha trabajado el tema de la filosofía [...] Nietzsche, sobre la perfección del hombre [...]". Jorge coincide en que trabajan "los hechos de la política, la política. [...] vimos la filosofía de algunos pensadores" (Grupo focal, 11 de diciembre 2014).

Los grupos coinciden en que los personajes que trabajan son principalmente hombres que han poseído poder político, económico, religioso o intelectual. Generalmente, ven a personajes particulares, como reyes, ministros, generales, filósofos, entre otros. Además, cuando esos personajes no están ligados a los temas mencionados, son trabajados como colectivos o 
cuasipersonajes, por ejemplo: los mapuches, el clero, el campesinado, entre otros. En efecto, se puede mencionar lo que comenta Juan, del grupo de Marcos, quien afirma que ven "por ejemplo, el señor feudal, el vasallo, y ahí se veía como un cierto grado de política, de las jerarquías, monarquías" (Grupo focal, 11 de diciembre 2014). Fabián, del mismo grupo, agrega que los temas que el profesor trata en clases son analizados desde las perspectivas políticas, económicas u otras que dan protagonismo a los hombres: "[...] generalmente en las clases del profesor, nos explica un cierto tema de la historia y después lo va analizando con los típicos tópicos, económica, política" (Grupo focal, 11 de diciembre).

Los jóvenes del grupo de Paula comentan que la tendencia en la enseñanza de la historia y las ciencias sociales es visibilizar y trabajar desde el protagonismo de los hombres que han tenido poder. Leo dice que son: "personajes políticos de la historia. En Roma los que los que mandaban $[\ldots]$ ". Mauricio agrega que suelen ser cuasipersonajes o grupos sociales con alta presencia masculina: "[...] emperadores [...], Julio César [...], Nobles". Según Richard: "en las clases de historia siempre vemos hombres" (Grupo focal, 4 de diciembre 2014). Por esta razón, Amelia, del grupo de Luis, critica y afirma que la poca presencia de las mujeres se debe a las jerarquías que existen sobre las acciones de las mujeres: “[...] solamente se habla más de hombres, porque como que el hombre es el 'todopoderoso'" (Grupo focal, 16 de diciembre 2014).

En este contexto, los participantes de todos los grupos coinciden en que las mujeres y su historia no son invisibles. No obstante, son trabajadas de forma esporádica y superficial. No se les da importancia ni protagonismo a sus acciones y su historia, siendo visibilizadas desde los estereotipos y los prejuicios por razones de género. Los integrantes del grupo de Marcos afirman que los hombres han construido imágenes basadas en estereotipos y prejuicios sobre las mujeres. En este sentido, los integrantes del grupo de Marcos comentan que han podido reflexionar sobre el rol que se les ha otorgado a los hombres en las clases de historia y ciencias sociales. Han concluido que ellos se han manifestado como los constructores de la historia, subordinando al resto de la población que no encaja con sus parámetros. Arturo se explica la marginación de las mujeres asumiendo que no son contenidos relevantes ni que vayan a ser profundizados en la enseñanza, debido a que "la mujer, en esos tiempos, cae en ese juego de que no es participe y al final se queda callada [...], la mujer es un punto aparte" (Grupo focal, 11 de diciembre 2014).

Los grupos de Paula y de Luis coinciden en que el protagonismo masculino por sobre el femenino ha sido provocado, entre otras razones, porque los hombres han escrito la historia $y$, por tanto, han marginado a las mujeres de las narrativas. Los jóvenes del grupo de Luis comentan que la historia no refleja: "ni el $5 \%$ de lo que debe haber pasado en relación

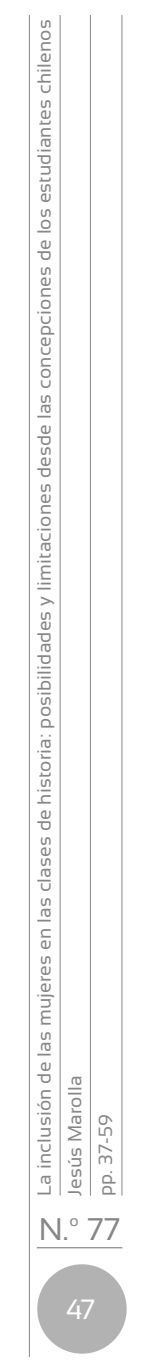


con las mujeres" (Gabriel). Amelia agrega que esto se debe a que el poder ha sido conservador por los hombres: "[...] los hombres siempre la han considerado como algo que 'sirve siempre para estar en la casa' [sic], o 'algo frágil'"' (Grupo focal, 16 de diciembre 2014).

Las razones que explican las breves apariciones que tienen las mujeres en las clases, tienen relación con la posición y el reconocimiento que la sociedad les ha dado. Las jóvenes del grupo de David comentan que las mujeres han realizado distintas acciones destacables durante la historia; no obstante, los hombres las han marginado a fin de que la sociedad las considere en una jerarquía inferior. Al respecto, Fany dice que debido a que es un país machista, se han generado subordinaciones hacia las mujeres donde se han visto relegadas a los espacios privados y las labores domésticas: "[...] creo que es porque es un país 'machista'. Entonces, ven al hombre como 'la fuerza" y es el que debe estar presente 'siempre'. Y a nosotras nos dejan como 'lavando los platos' [sic]" (Grupo focal, 9 de diciembre 2014).

Samuel, del grupo de Marcos esgrime las razones de la ausencia y la posición subordinada que se les dota a las mujeres: "no lo enseñan porque creen que la mujer es algo 'inferior' [...] la historia la basan solamente en los hombres y las cosas que hicieron las mujeres las dejan de lado solo porque son mujeres [sic], por eso no lo enseñan" (Grupo focal, 11 de diciembre 2014). Jorge y Arturo, del grupo de Marcos, coinciden en que la presencia marginada de las mujeres es debido a la construcción de la historia que han realizado los hombres. Asumen que es una construcción dominante presente en la sociedad: "[...] lo que muestran es que el hombre siempre va a ser el que descubre, va a ser como el 'protagonista' en la historia, nunca va a ser la mujer". (Arturo Grupo focal, 11 de diciembre 2014).

Fabián, del grupo de Marcos, plantea que la historia y la sociedad han construido una imagen y un rol de las mujeres como objeto, más que por su individualidad o por las acciones que realizan: "[...] como en casi todo el mundo lo más importante es el 'hombre'. Pero la mujer ¿en qué aparece? [...] en la mujer más bonita del mundo, en casi puros aspectos físicos para cumplir un cierto estándar creado por la humanidad" (Grupo focal, 11 de diciembre 2014). El grupo asume que los estereotipos, los prejuicios y la imagen pasiva y subordinada de las mujeres ha sido construida desde las jerarquías que han establecido los hombres, para así, validar sus acciones y poder.

Fany argumenta: "[...] en el libro de historia he encontrado dos planas que hablan de la mujer [...], la han omitido porque la mujer igual ha hecho 'hartas cosas' $[\mathrm{sic}]^{\prime \prime}$. La estudiante reconoce que las mujeres sí han realizado distintas acciones que han sido invisibilizadas debido a las estructuras androcéntricas de la sociedad: "[...] es un mundo machista [...], 
y no solo aquí en Chile, sino que en todos lados". Irma, por su parte, agrega que esto puede cambiar si es que se propone repensar la construcción de la historia, considerando las acciones de las mujeres: "[...] pero más que nada eso, el poder 'replantearse' todo lo que estamos viendo, el ver como otra cara de la misma cosa" (Grupo focal, 9 de diciembre 2014).

Como se ha expuesto, los grupos coinciden en que el machismo que existe en la sociedad es una de las estructuras que impide que la inclusión de las mujeres se valore y se le dé importancia a las acciones que han realizado en la construcción de la historia. Las jóvenes del grupo de David afirman que las mujeres que aparecen están subordinadas a lo que realizan los hombres. Critican la visión tradicional que se les ha dado a modo de anexo dentro de una estructura histórica dominada por el protagonismo de los hombres. Esas estructuras, comentan, transmiten un rol especifico basado en prejuicios y estereotipos. Camila afirma: "De hecho, queda hasta como un 'comodín'".

Los jóvenes del grupo de Marcos reflexionan sobre la falta de fomento del respeto y la tolerancia, ya que se considera a la mujer de manera inferior en contraste a los hombres. Los comentarios de Fabián y de Samuel de ese grupo agregan que no son solo las mujeres quienes han sido invisibilizadas en gran parte de la historia, sino que las diversidades de género son discriminadas en función de lo que establecen los hombres. Para Fabián, las razones son diversas: "[...] porque creo que se está viendo a la mujer o a otros tipos de sexualidad como algo 'inferior', como que muchas veces piensan que no importa si fue o no una mujer" (Grupo focal, 11 de diciembre 2014).

Para Fany, del grupo de David, la exclusión de las mujeres es provocada por las decisiones políticas tomadas por los hombres. Esto se debe a que, según la estudiante, si se incluyeran sus acciones y su participación en la construcción de la historia, ellas podrían generar empatía y, de esa manera, empoderarse en la sociedad. Se posicionarían en espacios dominados tradicionalmente por los hombres:

[...] si meten [sic] más a la mujer, estaría pucha [sic] más 'paros, tomas', [...] la juventud se va a "dar cuenta", nosotras también somos importantes y que necesitamos lo mismo, entonces, al Gobierno no le beneficia [...] que necesitan derechos, que necesitan la misma cantidad de sueldo que les dan a los hombres, entonces creo por eso siguen dejando a la mujer fuera. (Grupo focal, 9 de diciembre 2014).

Para Fany y sus compañeras, las acciones de las mujeres son tratadas de manera superficial, sin profundizar en las acciones que han realizado durante la historia ni en sus problemáticas desde una perspectiva de género. 


\section{Las ventajas y las dificultades de la inclusión de las mujeres y su historia en la enseñanza}

Los jóvenes afirman que las mujeres no están ausentes de las clases. El problema es el discurso bajo el cual se las visibiliza. Según los participantes, se fomenta la discriminación, la violencia y las conductas que las consideran en una situación de inferioridad social y en una jerarquía inferior en contraste a los espacios que ocupan los hombres. Coinciden que, en las escuelas, por lo general, las mujeres aparecen solo cuando se habla de la lucha por el derecho a sufragar. Los grupos plantean, incluso, que el profesorado se encarga de transmitir y perpetuar los estereotipos y los prejuicios de género a través de sus prácticas de aula.

Coinciden en asumir que una de las principales limitaciones para la inclusión de las mujeres en las clases es la sociedad androcéntrica y el machismo. Camila, del grupo de David, dice que: "[...] la cultura chilena así se ha criado [sic], con 'mentalidad machista'". Fany cuenta que desde las construcciones machistas se han tomado las decisiones de marginar su historia y acciones, por lo que las estudiantes creen que tales situaciones deberían transformarse: "[...] nunca hay como una participación de la mujer, siguen siendo machistas, siguen dejando a la 'mujer de lado' [...], hacen como que se vea 'lo mínimo' [sic]" (Grupo focal, 9 de diciembre 2014).

El grupo de Marcos agrega que, teniendo claro que la sociedad es machista y que dichas estructuras generan problemas sociales, el Ministerio no se ha encargado de plantear ni efectuar cambios en los libros o la enseñanza. Arturo dice que el Ministerio ha entregado un discurso que propone la inclusión y el respeto por la diversidad, no obstante, no fomentan una enseñanza que incluya a las mujeres y sus experiencias. Para él, las estructuras creadas por los hombres son rígidas y se basan en la tradicionalidad de la enseñanza, resistiendo el cambio: "[...] el Ministerio en ese sentido fue supercontradictorio, porque habla de que hay que respetar a la mujer pero al final no lo hace ni siquiera en sus propios libros" (Grupo focal, 11 de diciembre 2014).

Los grupos de Marcos, Luis y David coinciden en que una de las ventajas con la inclusión de las mujeres y su historia sería la posibilidad de crear modelos alternativos sobre los roles de las mujeres. Las jóvenes podrían identificarse con modelos alternativos a los tradicionales. A través de estos modelos y desde la problematización de las experiencias de las mujeres, los y las jóvenes podrían desarrollar empatía y comprometerse en acciones en contra de la discriminación y la desigualdad de género.

Las jóvenes del grupo de David plantean que debería incluirse a las mujeres a fin de que ellas pudieran conocer el protagonismo que han tenido, los roles que han desempeñado y así contar con modelos de 
identidad con los cuales empoderarse. Al respecto, Irma dice que podrían construir una identidad alternativa a la tradicional: "[...]creo que va en el conocimiento de identidad [...] saber quiénes somos nosotras y qué hicimos y qué se hizo" (Grupo focal, 9 de diciembre 2014).

Camila comenta que no se deben separar las experiencias de las mujeres de la historia general, ya que ambos contenidos colaboran en que ellas desde el empoderamiento puedan adoptar una posición para luchar contra las desigualdades sociales y de género: "[...] la historia de la mujer no tiene por qué ir aparte de la historia general. En sí, es exactamente lo mismo y te va a servir para lo mismo en el futuro [...] saber quién luchó por nuestros derechos" (Grupo focal, 9 de diciembre 2014).

Por último, los grupos de Marcos y de Luis coinciden en plantear como ventajas, la posibilidad de que se fomenten valores de tolerancia y de respeto ante la diversidad de género, la sexualidad y las libertades. Esto contribuiría a combatir el machismo que existe en la sociedad y del cual los grupos han coincidido en identificar como la principal estructura que genera discriminación, marginación y subordinación de género hacia las mujeres. Arturo, del grupo de Marcos, dice que la inclusión podría contribuir a que se desarrollaran valores de tolerancia hacia la diversidad de género. Reconoce que actualmente la discriminación es una realidad y, por tanto, hay que trabajar para cambiar tales problemas: "[...]creo que más que aprender de la mujer hay que aprender de todo tipo, como del pensamiento liberal, como la sexualidad, para ser una persona más tolerante" (Grupo focal, 11 de diciembre 2014).

\section{Discusión de los resultados}

Se desprende de los comentarios que han realizado los participantes que la enseñanza de la historia y de las ciencias sociales, tanto en las narrativas como en las prácticas del profesorado, más que cambiar las estructuras hegemónicas de género, las reproduce (Giroux, 1998). Por esto, los estudiantes hablan de tradición, entendida como una construcción hegemónica que varía muy poco (Apple, 1991). Esas construcciones han impedido que se incluyan mujeres (como otras perspectivas) y, más bien, han mantenido y replicado las estructuras históricas y sociales de género (Hidalgo, Dolores, Roset y Caba, 2003).

Siguiendo a Foucault (2008), la tradición podría ser el constructo bajo el cual se han validado y se han normalizado las estructuras sociales y las jerarquías que han generado las opresiones y las subyugaciones sociales. El conocimiento se entiende como externo a las personas y está controlado por quienes ostentan, definen y poseen el poder político, social y 
económico. La exclusión de las mujeres se explica debido a que la cultura hegemónica está compuesta y definida por los hombres y sus experiencias (Giroux, 1998).

Por lo anterior, los participantes plantean que la historia y las ciencias sociales se han construido a partir de estructuras hegemónicas patriarcales. Scott (2008) afirma que la historia se ha construido desde una jerarquía que ha posicionado el rol de los hombres como los protagonistas en una historia que los ha destacado como soldados, científicos y líderes, en oposición al rol subordinado que se les ha otorgado a las mujeres. El rol de las mujeres se ha presentado ligado a los planos privados y domésticos, coincidiendo con Levstik y Barton, (1997) y Pagès y Sant (2012), quienes aseguran que la historia ha sido construida bajo perspectivas que han marginado las experiencias de las mujeres y que las ha presentado de manera subordinada y secundaria a las acciones que han realizado los hombres.

Los participantes han afirmado que en las escuelas se fomenta una enseñanza de la historia que continúa reproduciendo las estructuras patriarcales de la sociedad. Los temas que se trabajan en la escuela tienen relación con la política, las guerras y la economía, ámbitos en los que el protagonismo lo tienen los hombres, mientras que los temas femeninos son considerados de segundo orden (Marolla y Pagès, 2015; Pagès y Sant, 2012).

El problema se hace latente cuando la perspectiva bajo la cual se visibiliza a las mujeres puede naturalizar la reproducción de la marginación, la subordinación, la desigualdad, los prejuicios y los estereotipos en torno a ellas (Scott, 2008). Hubbard (2013), Fernández (2006) y Vázquez (2003) coinciden con los estudiantes al considerar que las estructuras de género que se transmiten en las escuelas provocan que el discurso en la sociedad sobre los roles de las mujeres sea pasivo y esté subordinado a los roles dominantes de los hombres.

Visibilizar a las mujeres desde roles pasivos y subordinados provoca, como coinciden en afirmar los estudiantes, que problemáticas como la opresión, la discriminación y las jerarquías de género se produzcan y reproduzcan en la sociedad (Butler, 1999; Foucault, 2008; Wittig, 2006). Las jóvenes del grupo focal de David plantean que la normalización de las construcciones de género ha provocado que ellas no cuestionen la marginación y la subordinación de la que son parte. Se infiere que la desigualdad y la subordinación de los roles del ser mujer se han construido de manera normalizada, con el objetivo de naturalizar el silencio y la ausencia que se les ha impuesto en la historia.

Al respecto, los participantes coinciden en que se presenta un discurso normalizado sobre los roles de las mujeres que no incentiva ni llama a su participación en la sociedad. Por el contrario, busca la reproducción de las desigualdades de género. Esos problemas se podrían explicar desde lo 
que plantea Foucault (2005) como las estructuras de verdad en la historia, las cuales son construcciones culturales y de conocimiento que actúan como códigos culturales y de discursos que se transmiten a través de las técnicas, los valores, las jerarquías y las prácticas. De ahí que el poder se defina en términos de quiénes son los que controlan, difunden y producen tales códigos.

Los alumnos reconocen que los protagonistas de la historia con que trabajan están condicionados por las construcciones machistas de la sociedad, produciendo y entregando una imagen subordinada de las experiencias de las mujeres. Barton (2002) afirma que, aunque los estudiantes son conscientes de que los hombres y las mujeres han tenido diferentes experiencias en la historia, el discurso y el rol del que se apropian es el de una mujer que destaca por la pasividad y la no participación.

Giroux (2003) explica que la educación se ha construido posicionando los valores de los grupos dominantes (en este caso los hombres), provocando que no se cuestione la realidad y que se despoliticen los discursos y los códigos con que se trabajan en las aulas. Esto se relaciona con los comentarios de los jóvenes, donde afirmaban que la invisibilización de las mujeres y su historia provocaba que asumieran y se apropiaran de los roles pasivos y subordinados de ellas. Así los discursos y las estructuras que afirman la ausencia y la subordinación de las mujeres se consolidan. Más que destacar y problematizar las experiencias de las mujeres, se las presenta sin cuestionar las estructuras y los discursos de subordinación, marginación y discriminación (Scott, 2008)

Siguiendo a Spivak (1992), los participantes consideran que la visión de las mujeres se ha presentado, en general, desde la subalternidad y dentro de una construcción histórica que ha sido realizada por el patriarcado. Esto provoca, según la autora, que las mujeres hayan sido espectadoras de una historia que ha tenido como protagonistas a los hombres. Esto provoca que las alumnas construyan sus roles y sus identidades como mujeres que se asumen como ciudadanas, pero que no cuentan con las funciones que se les otorgan a los hombres. Asumen que la enseñanza y la visión de los roles de las mujeres son pasivas y están subordinadas a una estructura donde los hombres son los conductores de los procesos, y las mujeres, sus compañeras o espectadoras de estos.

En cuanto a las ventajas que conllevaría la inclusión de las mujeres y su historia, los estudiantes comentan que si se realiza desde una perspectiva crítica se podría favorecer el empoderamiento ciudadano, la participación activa y la identificación con roles de género alternativos a los tradicionales. Los distintos estudiantes de los grupos focales coinciden que se deberían problematizar los discursos sobre los roles y la participación de las mujeres. Banks (2004) y Crocco (2010) plantean, en coincidencia con 
los comentarios de los estudiantes, que para la identificación y el empoderamiento se deben exponer a reflexión las situaciones normalizadas y cotidianas de los prejuicios y la discriminación que han sufrido en el pasado como en el presente.

El hecho de que las mujeres sean visibilizadas como un apéndice en la enseñanza de la historia, provoca que los jóvenes no cuenten con modelos para emanciparse y luchar en contra de las desigualdades. Crocco (2010) y Scott (2008) coinciden con las jóvenes del grupo de David, quienes afirman que si se incluyeran mujeres desde perspectivas que problematizaran la ausencia y los discursos bajo los cuales se hacen visibles, ellas podrían contar con modelos que las alentarían desde la empatía hacia la participación. Se debe fomentar, según ellas, la reflexión y la crítica, a fin de comprender que la desigualdad, la diferencia y el silencio que se le ha otorgado a las mujeres, obedece a construcciones hegemónicas de género y de poder que pueden ser deconstruidas (Butler, 1999; Scott, 2008).

La problematización de las construcciones de la historia debería promover la búsqueda de respuestas y explicaciones a la ausencia y a los roles subordinados que se han transmitido sobre las mujeres en la enseñanza. La problematización, desde perspectivas críticas y reflexivas, debería promover que las chicas se empoderaran como actrices protagónicas en la sociedad, en los movimientos y en la lucha contra las desigualdades de género. De esta manera la enseñanza debe cuestionar la opresión de las mujeres tanto en el pasado como en el presente (Lerner, 1979), y cómo podría reconstruirse la historia considerando las experiencias de vida, las desigualdades y las posibilidades de emancipación (Butler, 1999) y participación en todos los espacios de la sociedad (Mcintosh, 1983).

Las jóvenes del grupo de David han afirmado que desde la problematización y la reconstrucción de las experiencias de las mujeres desde una perspectiva crítica, ellas podrían tomar conciencia de la situación de marginalidad y de opresión en que han vivido y así participar y luchar activamente en la sociedad. Esta opinión se podría relacionar con el concepto de Ross y Vinson (2006) de ciudadanía peligrosa. Manifiestan que el empoderamiento lo canalizarían a través de la protesta y la crítica a favor de la deconstrucción y la reconstrucción de los roles que les han impuesto.

Como han planteado los grupos, las mujeres y sus experiencias están excluidas de manera intencionada debido a que, si se reflexiona sobre la opresión, desde los gobiernos se tendrían que realizar modificaciones a las estructuras que han definido las subordinaciones y las jerarquías en torno a los géneros, y eso, para los participantes, significaría transformar las estructuras de poder (Foucault, 2008). 
El pensamiento crítico (Pagès y Sant, 2012) debe provocar que se cuestionen las construcciones discursivas que han normalizado y naturalizado las estructuras de género (Foucault, 2008), y de esa manera que las jóvenes, por ejemplo a través de la empatía (Heimberg, 2005) -entre otras opciones- se hagan y sientan parte de una historia que las ha invisibilizado, pero que empoderadas críticamente se pueden generar modelos que transformen las desigualdades por razones de género.

\section{Conclusiones}

En la investigación, los estudiantes han planteado distintos caminos, posibilidades y ventajas que se producirían con la inclusión de las mujeres y su historia. Afirman que el trabajo desde perspectivas críticas podría fomentar que ellos y ellas se empoderaran y participaran activamente con el objetivo de luchar contra las desigualdades de género. Los jóvenes reconocen los distintos problemas que se experimentan para incluir y problematizar las experiencias de las mujeres en las prácticas y en el proceso de enseñanza y aprendizaje. Agregan que su participación y acciones podrían contribuir a las transformaciones de género que se necesitan.

Las relaciones que se establecen en la escuela y en la enseñanza han sido construidas, según los comentarios de los grupos focales, desde perspectivas androcéntricas que han marginado a las mujeres. Esto provoca que no se establezcan espacios para la reflexión de las desigualdades de género ni se fomente la participación en la sociedad de las mujeres para luchar contra estos problemas. La ausencia y la subordinación que se les ha dotado, han provocado que estén marginadas no solo de las narrativas de la historia, sino que también de los espacios de la sociedad. De esa manera se continúan produciendo y reproduciendo las distintas jerarquías sociales y de género en la sociedad.

Si incluyeran las mujeres desde perspectivas que resaltaran sus acciones, podrían reflexionar sobre las construcciones patriarcales y las jerarquías. Ese es uno de los caminos para deconstruir las estructuras desiguales y así reconstruir los roles e identidades alternativas a las tradicionales. El fin debería ser el establecimiento de las posibilidades y del empoderamiento, la emancipación y la participación social crítica de las mujeres en la sociedad.

Aunque la enseñanza no ofrezca espacios para problematizar y criticar las construcciones actuales, los estudiantes afirman que las mujeres sí han realizado distintas acciones y que han sido marginadas debido a las decisiones de los hombres. Reconocen que están en una sociedad con distintas problemáticas y desigualdades de género. Plantean que, para revertir y luchar contra dichos problemas, se deberían problematizar y 
hacer visibles, desde la educación, las construcciones actuales sobre la historia y su enseñanza.

Los estudiantes plantean que la enseñanza debe posibilitar los espacios reflexivos y críticos que cuestionen las construcciones que han marginado a las personas por poseer una identidad distinta a las tradicionales. El objetivo, siguiendo a los participantes, no es tematizar ni trabajar en cursos anexos los problemas de las mujeres a modo de relato histórico, sino que se les entreguen los espacios de crítica a fin de reflexionar de forma transversal sobre el sistema que ha producido y reproducido las problemáticas y las injusticias.

Por último, los alumnos dicen que la enseñanza no solo se debe remitir a la transmisión de contenidos, sino que debe comenzar a transformar los espacios educativos a fin de que la inclusión de las diferencias posibilite la participación. La educación y la enseñanza de la historia debe tener como fin problematizar las construcciones de género desiguales y exponer el poder y las jerarquías tradicionales que han poseído los hombres por sobre las mujeres. El objetivo debería ser que la enseñanza se enfocara en la justicia social y el reconocimiento de la diversidad de género.

\section{Referencias}

Álvarez-Gayou, J.L. (2003). Cómo hacer investigación cualitativa. Fundamentos y metodología. México: Paidós.

Apple, M. (1991). Ideología y currículo. Madrid: Akal.

Banks, J.A. (2004). Introduction: Democratic Citizenship Education in a Multicultural Societies. En J.A. Banks, Diversity and Citizenship Education (pp. 3-16). San Francisco: Jossey-Bass.

Bardin, L. (1986). El análisis de contenido. Madrid: Ediciones Akal.

Barton, K. (2002). Masculinity and Schooling. Theory and Research in Social Education, 30(2), 306-312.

Bisquerra, R. (2004). Metodología de la investigación educativa. Madrid: La Muralla.

Britzman, D. (2002). La pedagogía transgresora y sus extrañas técnicas. En R. Mérida (ed.), Sexualidades transgresoras. Una antología de estudios queer (pp. 197-228). Barcelona: Icaria.

Butler, J. (1999). El género en disputa. El feminismo y la subversión de la identidad. México: Paidós.

Butler, J. (2001). Mecanismos psíquicos del poder: teorías sobre la sujeción. Madrid: Ediciones Cátedra.

Butler, J. (2006). Deshacer el género. Barcelona: Paidós Ibérica.

Cohen, L., Manion, L. y Morrison, K. (2007). Research Methods in Education. Londres: Routledge. 
Creswell, J. (2014). Research design. Qualitative, Quantitative and Mixed Methods Approaches. California: Sage.

Crocco, M.S. (2006). Gender and social education: What's the problem? En E.W. Ross (ed.), The social studies curriculum: Purposes, problems, and possibilities (pp. 171-193). Albany: State University of New York Press.

Crocco, M.S. (2010). Using Literature to Teach about Others, the case of Shabanu. En W. (2008). Gender and Sexuality in Social Studies. En L. Levstik, C. Tyson, Handbook of Research in Social Studies Education (pp. 172-196). Nueva York: Routledge.

Crocco, M.S. Parker, Social Studies Today: Research and Practice (pp. 175182). Nueva York: Routledge.

Fernández, A. (2006). La construcción de identidad desde la perspectiva de Género. Íber, 47, 33-44.

Finlay, L. (2002). Negotiating the swamp: the opportunity and challenge of reflexivity in research practice. Qualitative Research, 2(2), 209-230.

Foucault, M. (2005). Vigilar y castigar. Nacimiento de la prisión. Madrid: Siglo xxı.

Foucault, M. (2008). Historia de la sexualidad 1: la voluntad del saber. Buenos Aires: Siglo xxı.

Giroux, H. (1998). Las políticas de educación y cultura. En H. Giroux y P. Mclaren, Sociedad, cultura y educación (pp. 79-86). Madrid: Niño y Dávila Editores.

Giroux, H. (2003). Pedagogía y política de la esperanza. Teoría, cultura y enseñanza. Madrid: Amorrortu Editores.

Habermas, J. (1988). La lógica de las Ciencias Sociales. Madrid: Tecnos.

Heimberg, Ch. (2005). L'alterité et le multiculturalismo au coeur de I'histoire enseignée. En C. García Ruiz et al. (eds.), Enseñar ciencias sociales en una sociedad multicultural (pp. 17-32). Almería: Universidad de Almería.

Hidalgo, E., Dolores, J., Roset, M. y Caba, Á. (2003). Repensar la enseñanza de la geografía y la historia. Una mirada desde el género. Barcelona: Octaedro.

Hubbard, P. (2013). Kissing is not a universal right: Sexuality, law and the scales of citizenship. Geoforum, 49, 224-232.

Lather, P. (1992). Post-critical pedagogies: a feminist reading. En C. Luke y J. Gore (ed.), Feminisms and critical pedagogy. Nueva York: Routledge.

Lerner, G. (1979). The Majority Finds it Past: Placing Women in History. Nueva York: Oxford University Press.

Levstik, L. y Barton, K. (1997). Doing history: Investigating with children in elementary and middle schools. Mahwah, NJ: Lawrence Erlbaum Associates, Publishers. 
Levstik, L. y Groth, J. (2002). Scary thing being an eighth grader: Exploring gender and sexuality in a middle school US history unit. Theory and Research in Social Education, 30(2), 233-254.

Lomas, C. (2002). El sexismo en los libros de texto. En A. González y C. Lomas (coords.), Mujer y educación (pp. 193-209). Barcelona: Graó.

Macbeth, D. (2001). Reflexivity in Qualitative Research: Two Readings, and a Third. Qualitative Inquiry, 7(35), 35-68.

Marolla, J. y Pagès, J. (2015). Ellas sí tienen historia. Las representaciones del profesorado chileno de secundaria sobre la enseñanza de la historia de las mujeres. Clío \& Asociados, 21, 223-236.

Mcintosh, P. (1983). Interactive phases of curricular re-vision. A Feminist Perspective. MA: Wellesley College.

Miles, M. y Huberman, M. (1994). Qualitative Data Analysis: A Sourcebook of New Methods. Thousand Oaks, CA: Sage.

Moreno, M. y Sastre, G. (2003). Visión de la enseñanza desde otra identidad. En M.D. Villuendas y J. Gordo López (eds.), Relaciones de género en psicología y educación (pp. 65-76). Madrid: Comunidad de Madrid.

Mouffe, Ch. (1993). Feminismo, ciudadanía y política democrática radical. Debate Feminista, 4(7), 3-22.

Oakley, A. (1998). Gender, methodology and people's ways of knowing: some problems with Feminism and the paradigm debate in Social Science. Sociology, 32(4), 707-731.

Pagés, J. y Sant, E. (2012) Las mujeres en la enseñanza de la Historia: ¿Hasta cuándo serán invisibles? Cad. Pesq. CDhis, 25(1), 91-117.

Ross, W. y Vinson, K. (2006). La educación para una ciudadanía peligrosa. Enseñanza de las Ciencias Sociales, 11, 73-86.

Scott, J.W. (2008). Género e historia. México: Fondo de Cultura Económica.

Simons, H. (2011). El estudio de caso: Teoría y práctica. Madrid: Morata.

Spivak, G. (2007). French Feminism Revisited: Ethics and Politics. En J. Butler y J. W. Scott (eds.), Feminist Theorize the Political (pp. 54-85). Nueva York: Routledge.

Stake, R.E. (2007). Investigación con estudio de casos. Madrid: Morata.

Subirats, M. (2001). ¿Qué es educar? De la necesidad de reproducción a la necesidad de cambio. En A. Tomé y X. Rambla, Contra el sexismo: coeducación y democracia en la escuela (pp. 17-21). Madrid: Síntesis.

Talburt, S. y Steinberg, S. (2005). Pensando queer. Sexualidad, cultura y educación. Barcelona: Graó.

Usher, P. (1996). Feminist approaches to research. En D. Scott y R. Usher (eds.), Understanding Educational Research (pp. 120-142). Londres: Routledge. 
Vázquez, X. (2003). Educación e igualdad de oportunidades entre sexos. Barcelona: ICE-Horsori.

Wittig, M. (2006). El pensamiento heterosexual y otros ensayos. Barcelona: Egales.

Wolcott, H. (1994). Transforming Qualitative Data: Description, Analysis and Interpretation. Londres: Sage.

Woyshner, C. (2002). Political History as Women's History: Toward a More Inclusive Curriculum. Theory and Research in Social Education, 30(3), 354-380.

Yin, R. (2009). Case Study Research: Design and Methods. Thousand Oaks, CA: Sage. 